\title{
Boundary value problems for Caputo fractional differential equations with nonlocal and fractional integral boundary conditions
}

Received: 12 January 2019 / Accepted: 11 July 2020 / Published online: 22 July 2020

(C) The Author(s) 2020

\begin{abstract}
In this paper, we study the existence and uniqueness of solutions for fractional differential equations with nonlocal and fractional integral boundary conditions. New existence and uniqueness results are established using the Banach contraction principle. Other existence results are obtained using O'Regan fixed point theorem and Burton and Kirk fixed point. In addition, an example is given to demonstrate the application of our main results.
\end{abstract}

Mathematics Subject Classification $34 \mathrm{~A} 08 \cdot 26 \mathrm{~A} 33$

\section{Introduction}

Fractional differential equations have gained much importance and attention due to the fact that they have been proved to be valuable tools in the modeling of many phenomena in engineering and sciences such as physics, mechanics, economics, biology, etc. (see, for example, $[16,19,23,24]$ and references therein). Actually, the concepts of fractional derivatives are not the only generalization of the ordinary derivatives, but also it has been found that they can efficiently and properly describe the behavior of many physical systems (real-life phenomena) more accurately than integer-order derivatives.

Different kind of fixed point theorems are widely used as fundamental tools to prove the existence and uniqueness of solutions for various classes of fractional differential equations; for example, we refer the reader to $[1-4,9,12,14,15,17,18,21,25,27]$ and the references cited therein. Moreover, some mathematicians considered Caputo fractional differential equations with a nonlinear term depending on the Caputo derivative (see Benchohra and Souid [5], Benchohra and Lazreg [6], Benchohra et al. [7], El-Sayed and Bin-Taher [10, 11], Guezane-Lakoud and Khaldi [12], Guezane-Lakoud and Bensebaa [13], Houas and Benbachir [14], Nieto et al. [20], and Yan et al. [27]).

Motivated by the above papers, in this paper, we establish various existence and uniqueness results of solutions for a boundary value problem of nonlinear fractional differential equations of order $\alpha \in(2,3]$ with nonlocal and fractional integral boundary conditions given by:

$$
\begin{cases}{ }^{c} D_{0^{+}}^{\alpha} u(t)=f\left(t, u(t),{ }^{c} D_{0^{+}}^{\beta} u(t)\right), t \in J:=[0,1] & \\ u(0)=g(u), & 0<\eta_{1}<1 \\ u^{\prime}(0)=a I_{0^{+}}^{\sigma_{1}} u\left(\eta_{1}\right), & 0<\eta_{2}<1, \\ { }^{c} D_{0^{+}}^{\beta_{1}} u(1)=b I_{0^{+}}^{\sigma_{2}} u\left(\eta_{2}\right), & \end{cases}
$$

C. Derbazi $(\varangle)$, H. Hammouche

Laboratory of Mathematics and Applied Sciences, University of Ghardaia, 47000 Metlili, Algeria

E-mail: choukriedp@yahoo.com

E-mail: h.hammouche@yahoo.fr 
where ${ }^{c} D_{0^{+}}^{\kappa}$ is the Caputo fractional derivative of order $\kappa \in\left\{\alpha, \beta, \beta_{1}\right\}$, such that $2<\alpha \leq 3$, and $0<\beta, \beta_{1} \leq 1$, and $f:[0,1] \times \mathbb{R}^{2} \longrightarrow \mathbb{R}$ and $g: C([0,1], \mathbb{R}) \longrightarrow \mathbb{R}$ are continuous functions, $I_{0^{+}}^{\sigma_{i}}$ is the Riemann-Liouville fractional integral of order $\sigma_{i}>0, i=1,2, \eta_{1}, \eta_{2}, a, b$ are suitably chosen real constants, such that:

$$
\begin{aligned}
\Delta= & {\left[\left(\frac{a \eta_{1}^{\sigma_{1}+1}}{\Gamma\left(\sigma_{1}+2\right)}-1\right)\left(\frac{b \eta_{2}^{\sigma_{2}+2}}{\Gamma\left(\sigma_{2}+3\right)}-\frac{1}{\Gamma\left(3-\beta_{1}\right)}\right)\right.} \\
& \left.-\left(\frac{b \eta_{2}^{\sigma_{2}+1}}{\Gamma\left(\sigma_{2}+2\right)}-\frac{1}{\Gamma\left(2-\beta_{1}\right)}\right)\left(\frac{a \eta_{1}^{\sigma_{1}+2}}{\Gamma\left(\sigma_{1}+3\right)}\right)\right] \neq 0 .
\end{aligned}
$$

The paper is organized as follows. In Sect. 2, we collect some background material and basic results about fractional calculus that will be used later. In Sect. 3, we establish the existence of solutions for the boundary value problem (1) using Banach contraction principle, O'Regan fixed point theorem, and Burton and Kirk fixed point. In the fourth section, we give an example to demonstrate the application of our main results. Finally, we add a conclusion to the paper in the last section.

\section{Preliminaries}

In this section, we will recall some notions and definition which we will need in the sequel (details can be found, e.g., in $[16,23,24])$.

Definition 2.1 ([16]) The Riemann-Liouville fractional integral of order $\alpha>0$ of a function $u \in L^{1}([0,1])$ is defined by:

$$
I_{0^{+}}^{\alpha} u(t)=\frac{1}{\Gamma(\alpha)} \int_{0}^{t}(t-s)^{\alpha-1} u(s) \mathrm{d} s, \quad(t>0, \alpha>0)
$$

where $\Gamma(\cdot)$ is the (Euler's) Gamma function $\Gamma(\alpha)=\int_{0}^{+\infty} e^{-t} t^{\alpha-1} \mathrm{~d} t, \quad \alpha>0$. Moreover, for $\alpha=0$, we set $I_{0^{+}}^{0} u:=u$.

Definition 2.2 ([16,23]) The Caputo fractional derivative of order $\alpha$ of a function $u \in A C^{n}([0,1])$ is represented by:

$$
{ }^{c} D_{0^{+}}^{\alpha} u(t)= \begin{cases}\frac{1}{\Gamma(n-\alpha)} \int_{0}^{t}(t-s)^{n-\alpha-1} u^{(n)}(s) \mathrm{d} s, & \text { if } \alpha \notin \mathbb{N}, \\ u^{(n)}(t), & \text { if } \alpha \in \mathbb{N},\end{cases}
$$

where $u^{(n)}(t)=\frac{\mathrm{d}^{n} u(t)}{\mathrm{d} t^{n}}, \alpha>0, n=[\alpha]+1$ and $[\alpha]$ denotes the integer part of the real number $\alpha$.

Lemma 2.3 Let $\alpha, \beta>0, n=[\alpha]+1$, and then, the following relation holds:

$$
{ }^{c} D_{0^{+}}^{\alpha} t^{\beta}= \begin{cases}\frac{\Gamma(\beta+1)}{\Gamma(\beta-\alpha+1)} t^{\beta-\alpha}, & (\beta \in \mathbb{N} \text { and } \beta \geq n \text { or } \beta \notin \mathbb{N} \text { and } \beta>n-1), \\ 0, & \beta \in\{0, \ldots, n-1\} .\end{cases}
$$

Lemma 2.4 Let $\alpha>\beta>0$, and $u \in L^{1}([0,1])$. Then, we have:

(1) $I_{0^{+}}^{\alpha} I_{0^{+}}^{\beta} u(t)=I_{0^{+}}^{\alpha+\beta} u(t)$,

(2) ${ }^{c} D_{0^{+}}^{\alpha} I_{0^{+}}^{\alpha} u(t)=u(t)$,

(3) ${ }^{c} D_{0^{+}}^{\beta} I_{0^{+}}^{\alpha} u(t)=I_{0^{+}}^{\alpha-\beta} u(t)$. 
Lemma 2.5 Let $\alpha>0$. Then, the differential equation

$$
\left({ }^{c} D_{0^{+}}^{\alpha} u\right)(t)=0,
$$

has solutions

$$
u(t)=\sum_{j=0}^{n-1} c_{j} t^{j}, \quad c_{j} \in \mathbb{R}, j=0 \ldots n-1,
$$

where $n-1<\alpha<n$

Lemma 2.6 Let $\alpha>0$. Then:

$$
I_{0^{+}}^{\alpha}\left({ }^{c} D_{0^{+}}^{\alpha} u(t)\right)=u(t)+\sum_{j=0}^{n-1} c_{j} t^{j},
$$

for some $c_{j} \in \mathbb{R}, j=0,1,2, \ldots, m-1$, where $n=[\alpha]+1$.

Lemma 2.7 Let $\alpha>0, u \in L^{1}([0,1], \mathbb{R})$. Then, for all $t \in[0,1]$, we have:

$$
I_{0^{+}}^{\alpha+1} u(t) \leq\left\|I_{0^{+}}^{\alpha} u\right\|_{L^{1}} .
$$

Proof Let $u \in L^{1}([0,1], \mathbb{R})$, and from Lemma 2.4, we have:

$$
I_{0^{+}}^{\alpha+1} u(t)=I_{0^{+}}^{1} I_{0^{+}}^{\alpha} u(t)=\int_{0}^{t} I_{0^{+}}^{\alpha} u(s) d s \leq \int_{0}^{1}\left|I_{0^{+}}^{\alpha} u(s)\right| \mathrm{d} s=\left\|I_{0^{+}}^{\alpha} u\right\|_{L^{1}} .
$$

Lemma 2.8 The fractional integral $I_{0^{+}}^{\alpha}, \alpha>0$ is bounded in $L^{1}([0,1], \mathbb{R})$ with

$$
\left\|I_{0^{+}}^{\alpha} u\right\|_{L^{1}} \leq \frac{\|u\|_{L^{1}}}{\Gamma(\alpha+1)}
$$

Proof Let $u \in L^{1}([0,1], \mathbb{R})$, and then:

$$
\begin{aligned}
\left\|I_{0^{+}}^{\alpha} u\right\|_{L^{1}} & =\int_{0}^{1}\left|I_{0^{+}}^{\alpha} u(t)\right| \mathrm{d} t \leq \frac{1}{\Gamma(\alpha)} \int_{0}^{1} \int_{0}^{t}(t-s)^{\alpha-1}|u(s)| \mathrm{d} s \mathrm{~d} t \\
& \leq \frac{1}{\Gamma(\alpha)} \int_{0}^{1}|u(s)| \mathrm{d} s \int_{s}^{1}(t-s)^{\alpha-1} \mathrm{~d} t \\
& \leq \frac{1}{\Gamma(\alpha+1)} \int_{0}^{1}(t-s)^{\alpha}|u(s)| \mathrm{d} s \\
& \leq \frac{\|u\|_{L^{1}}}{\Gamma(\alpha+1)}
\end{aligned}
$$

Let us now introduce the space $E=\left\{u: u \in C([0,1], \mathbb{R}):{ }^{c} D_{0^{+}}^{\beta} u \in C([0,1], \mathbb{R})\right\}$ equipped with the norm:

$$
\|u\|_{E}=\|u\|_{\infty}+\left\|{ }^{c} D_{0^{+}}^{\beta} u\right\|_{\infty}=\sup _{t \in J}|u(t)|+\sup _{t \in J}\left|{ }^{c} D_{0^{+}}^{\beta} u(t)\right| .
$$

Clearly, $\left(E,\|\cdot\|_{E}\right)$ is a Banach space [26]. 


\section{Main results}

For the existence of solutions for the problem (1), we need the following lemma:

Lemma 3.1 For a given $h \in C(J, \mathbb{R})$, the unique solution of the linear fractional boundary value problem:

$$
{ }^{c} D_{0^{+}}^{\alpha} u(t)=h(t), 2<\alpha \leq 3,
$$

supplemented with boundary conditions

$$
u(0)=g(u), \quad u^{\prime}(0)=a I_{0^{+}}^{\sigma_{1}} u\left(\eta_{1}\right), \quad{ }^{c} D_{0^{+}}^{\beta_{1}} u(1)=b I_{0^{+}}^{\sigma_{2}} u\left(\eta_{2}\right),
$$

is given by:

$$
\begin{aligned}
u(t)= & I_{0^{+}}^{\alpha} h(t)+\left(v_{6} t^{2}-v_{1} t\right) I_{0^{+}}^{\alpha+\sigma_{1}} h\left(\eta_{1}\right)+\left(v_{2} t-v_{5} t^{2}\right) I_{0^{+}}^{\alpha+\sigma_{2}} h\left(\eta_{2}\right) \\
& +\left(v_{8} t^{2}-v_{4} t\right) I_{0^{+}}^{\alpha-\beta_{1}} h(1)+\left(1-v_{3} t-v_{7} t^{2}\right) g(u),
\end{aligned}
$$

where

$$
\begin{aligned}
& v_{1}=\frac{a}{\Delta}\left(\frac{b \eta_{2}^{\sigma_{2}+2}}{\Gamma\left(\sigma_{2}+3\right)}-\frac{1}{\Gamma\left(3-\beta_{1}\right)}\right), v_{2}=\frac{a b}{\Delta}\left(\frac{\eta_{1}^{\sigma_{1}+2}}{\Gamma\left(\sigma_{1}+3\right)}\right), \\
& v_{3}=\frac{\eta_{1}^{\sigma_{1}} v_{1}}{\Gamma\left(\sigma_{1}+1\right)}-\frac{\eta_{2}^{\sigma_{2}} v_{2}}{\Gamma\left(\sigma_{2}+1\right)}, v_{4}=\frac{v_{2}}{b}, v_{5}=\frac{b}{2 \Delta}\left(\frac{a \eta_{1}^{\sigma_{1}+1}}{\Gamma\left(\sigma_{1}+2\right)}-1\right), \\
& v_{6}=\frac{a}{2 \Delta}\left(\frac{b \eta_{2}^{\sigma_{2}+1}}{\Gamma\left(\sigma_{2}+2\right)}-\frac{1}{\Gamma\left(2-\beta_{1}\right)}\right), v_{7}=\frac{\eta_{2}^{\sigma_{2}} v_{5}}{\Gamma\left(\sigma_{2}+1\right)}-\frac{\eta_{1}^{\sigma_{1}} v_{6}}{\Gamma\left(\sigma_{1}+1\right)}, \\
& v_{8}=\frac{v_{5}}{b}
\end{aligned}
$$

and $\Delta$ is defined by (2).

Proof Applying the Riemann-Liouville fractional integral operator of order $\alpha$ to both sides of (3) and using Lemma 2.6, we have:

$$
u(t)=I_{0^{+}}^{\alpha} h(t)-c_{0}-c_{1} t-c_{2} t^{2}, \quad c_{0}, c_{1}, c_{2} \in \mathbb{R} .
$$

Applying the boundary conditions (4) in (7), we find that:

$$
c_{0}=-g(u) .
$$

Using the boundary conditions of (4) in (7), we find a system of equations:

$$
\begin{aligned}
& \left(\frac{a \eta_{1}^{\sigma_{1}+1}}{\Gamma\left(\sigma_{1}+2\right)}-1\right) c_{1}+2\left(\frac{a \eta_{1}^{\sigma_{1}+2}}{\Gamma\left(\sigma_{1}+3\right)}\right) c_{2}=a I_{0^{+}}^{\alpha+\sigma_{1}} h\left(\eta_{1}\right)+\left(\frac{a \eta_{1}^{\sigma_{1}}}{\Gamma\left(\sigma_{1}+1\right)}\right) g(u) . \\
& \left(\frac{b \eta_{2}^{\sigma_{2}+1}}{\Gamma\left(\sigma_{2}+2\right)}-\frac{1}{\Gamma\left(2-\beta_{1}\right)}\right) c_{1}+2\left(\frac{b \eta_{2}^{\sigma_{2}+2}}{\Gamma\left(\sigma_{2}+3\right)}-\frac{1}{\Gamma\left(3-\beta_{1}\right)}\right) c_{2}=b I_{0^{+}}^{\alpha+\sigma_{2}} h\left(\eta_{2}\right) \\
& +\left(\frac{b \eta_{2}^{\sigma_{2}}}{\Gamma\left(\sigma_{2}+1\right)}\right) g(u)-I_{0^{+}}^{\alpha-\beta_{1}} h(1) .
\end{aligned}
$$

Solving the system of equations (8) and (9) together with notations (6), we find that:

$$
\begin{aligned}
& c_{1}=v_{1} I_{0^{+}}^{\alpha+\sigma_{1}} h\left(\eta_{1}\right)-v_{2} I_{0^{+}}^{\alpha+\sigma_{2}} h\left(\eta_{2}\right)+v_{3} g(u)+v_{4} I_{0^{+}}^{\alpha-\beta_{1}} h(1), \\
& c_{2}=v_{5} I_{0^{+}}^{\alpha+\sigma_{2}} h\left(\eta_{2}\right)-v_{6} I_{0^{+}}^{\alpha+\sigma_{1}} h\left(\eta_{1}\right)+v_{7} g(u)-v_{8} I_{0^{+}}^{\alpha-\beta_{1}} h(1) .
\end{aligned}
$$

Substituting the values of $c_{0}, c_{1}, c_{2}$ in (7), we obtain the solution (5). This completes the proof. 
For computational convenience, we introduce the notations:

$$
I_{0^{+}}^{\alpha} f\left(s, u(s),{ }^{c} D_{0^{+}}^{\beta} u(s)\right)(t)=\frac{1}{\Gamma(\alpha)} \int_{0}^{t}(t-s)^{\alpha-1} f\left(s, u(s),{ }^{c} D_{0^{+}}^{\beta} u(s)\right) \mathrm{d} s, \quad \forall t \in J .
$$

Define the integral operator $T: E \longrightarrow E$ by:

$$
\begin{aligned}
T(u)(t)= & I_{0^{+}}^{\alpha} f\left(s, u(s),{ }^{c} D_{0^{+}}^{\beta} u(s)\right)(t) \\
& +\left(v_{6} t^{2}-v_{1} t\right) I_{0^{+}}^{\alpha+\sigma_{1}} f\left(s, u(s),{ }^{c} D_{0^{+}}^{\beta} u(s)\right)\left(\eta_{1}\right) \\
& +\left(v_{2} t-v_{5} t^{2}\right) I_{0^{+}}^{\alpha+\sigma_{2}} f\left(s, u(s),{ }^{c} D_{0^{+}}^{\beta} u(s)\right)\left(\eta_{2}\right) \\
& +\left(v_{8} t^{2}-v_{4} t\right) I_{0^{+}}^{\alpha-\beta_{1}} f\left(s, u(s),{ }^{c} D_{0^{+}}^{\beta} u(s)\right)(1) \\
& +\left(1-v_{3} t-v_{7} t^{2}\right) g(u) .
\end{aligned}
$$

Next, we introduce two operators $T_{1,2}: E \longrightarrow E$ as follows:

$$
\begin{aligned}
T_{1}(u)(t)= & I_{0^{+}}^{\alpha} f\left(s, u(s),{ }^{c} D_{0^{+}}^{\beta} u(s)\right)(t) \\
& +\left(v_{6} t^{2}-v_{1} t\right) I_{0^{+}}^{\alpha+\sigma_{1}} f\left(s, u(s),{ }^{c} D_{0^{+}}^{\beta} u(s)\right)\left(\eta_{1}\right) \\
& +\left(v_{2} t-v_{5} t^{2}\right) I_{0^{+}}^{\alpha+\sigma_{2}} f\left(s, u(s),{ }^{c} D_{0^{+}}^{\beta} u(s)\right)\left(\eta_{2}\right) \\
& +\left(v_{8} t^{2}-v_{4} t\right) I_{0^{+}}^{\alpha-\beta_{1}} f\left(s, u(s),{ }^{c} D_{0^{+}}^{\beta} u(s)\right)(1),
\end{aligned}
$$

and

$$
T_{2} u(t)=\left(1-v_{3} t-v_{7} t^{2}\right) g(u)
$$

Clearly:

$$
T u(t)=T_{1} u(t)+T_{2} u(t), t \in J .
$$

Remark 3.2 In our work, we assume that the restriction $\Delta \neq 0$ is satisfied, because in the case when $\Delta=0$, the problem (1) will be under the resonant conditions, and hence, the previously mentioned theorems are not applicable, and then, the problem can be managed using the method of Mawhin continuation theorem.

Lemma 3.3 Let $f \in C([0,1] \times \mathbb{R} \times \mathbb{R}, \mathbb{R})$. Then, $u \in E$ is a solution of the fractional boundary value problem (1) if and only if $T u(t)=u(t), \forall t \in[0,1]$.

Proof Let $u$ be a solution of (1). Then, using the same method as used in Lemma 3.1, we can prove that:

$$
\begin{aligned}
u(t)= & T(u)(t)=I_{0^{+}}^{\alpha} f\left(s, u(s),{ }^{c} D_{0^{+}}^{\beta} u(s)\right)(t) \\
& +\left(v_{6} t^{2}-v_{1} t\right) I_{0^{+}}^{\alpha+\sigma_{1}} f\left(s, u(s),{ }^{c} D_{0^{+}}^{\beta} u(s)\right)\left(\eta_{1}\right) \\
& +\left(v_{2} t-v_{5} t^{2}\right) I_{0^{+}}^{\alpha+\sigma_{2}} f\left(s, u(s),{ }^{c} D_{0^{+}}^{\beta} u(s)\right)\left(\eta_{2}\right) \\
& +\left(v_{8} t^{2}-v_{4} t\right) I_{0^{+}}^{\alpha-\beta_{1}} f\left(s, u(s),{ }^{c} D_{0^{+}}^{\beta} u(s)\right)(1) \\
& +\left(1-v_{3} t-v_{7} t^{2}\right) g(u) .
\end{aligned}
$$

Conversely, $u$ satisfies:

$$
\begin{aligned}
u(t)= & I_{0^{+}}^{\alpha} f\left(s, u(s),{ }^{c} D_{0^{+}}^{\beta} u(s)\right)(t) \\
& +\left(v_{6} t^{2}-v_{1} t\right) I_{0^{+}}^{\alpha+\sigma_{1}} f\left(s, u(s),{ }^{c} D_{0^{+}}^{\beta} u(s)\right)\left(\eta_{1}\right) \\
& +\left(v_{2} t-v_{5} t^{2}\right) I_{0^{+}}^{\alpha+\sigma_{2}} f\left(s, u(s),{ }^{c} D_{0^{+}}^{\beta} u(s)\right)\left(\eta_{2}\right) \\
& +\left(v_{8} t^{2}-v_{4} t\right) I_{0^{+}}^{\alpha-\beta_{1}} f\left(s, u(s),{ }^{c} D_{0^{+}}^{\beta} u(s)\right)(1) \\
& +\left(1-v_{3} t-v_{7} t^{2}\right) g(u),
\end{aligned}
$$


and denotes the right-hand side of the equation by $v(t)$. Then, by Lemma 2.4, we obtain:

$$
\begin{aligned}
{ }^{c} D_{0^{+}}^{\alpha} v(t)= & { }^{c} D_{0^{+}}^{\alpha} I_{0^{+}}^{\alpha} f\left(s, u(s),{ }^{c} D_{0^{+}}^{\beta} u(s)\right)(t) \\
& +{ }^{c} D_{0^{+}}^{\alpha}\left(v_{6} t^{2}-v_{1} t\right) I_{0^{+}}^{\alpha+\sigma_{1}} f\left(s, u(s),{ }^{c} D_{0^{+}}^{\beta} u(s)\right)\left(\eta_{1}\right) \\
& +{ }^{c} D_{0^{+}}^{\alpha}\left(v_{2} t-v_{5} t^{2}\right) I_{0^{+}}^{\alpha+\sigma_{2}} f\left(s, u(s),{ }^{c} D_{0^{+}}^{\beta} u(s)\right)\left(\eta_{2}\right) \\
& +{ }^{c} D_{0^{+}}^{\alpha}\left(v_{8} t^{2}-v_{4} t\right) I_{0^{+}}^{\alpha-\beta_{1}} f\left(s, u(s),{ }^{c} D_{0^{+}}^{\beta} u(s)\right)(1) \\
& +{ }^{c} D_{0^{+}}^{\alpha}\left(1-v_{3} t-v_{7} t^{2}\right) g(u) \\
= & f\left(t, u(t),{ }^{c} D_{0^{+}}^{\beta} u(t)\right) .
\end{aligned}
$$

Hence, $v(t)$ is a solution of the fractional differential Eq. (1). Also, it is easy to verify by direct computation that $v$ satisfies conditions (4). This completes the proof.

To simplify the computations in the main results, we set some notations:

$$
\begin{aligned}
M_{1}= & \frac{1}{\Gamma(\alpha)}+\frac{\left|v_{6}\right|+\left|v_{1}\right|}{\Gamma\left(\alpha+\sigma_{1}\right)}+\frac{\left|v_{2}\right|+\left|v_{5}\right|}{\Gamma\left(\alpha+\sigma_{2}\right)}+\frac{\left|v_{8}\right|+\left|v_{4}\right|}{\Gamma\left(\alpha-\beta_{1}\right)}, \\
M_{2}= & \frac{1}{\Gamma(\alpha-\beta)}+\frac{2\left|v_{6}\right|+(2-\beta)\left|v_{1}\right|}{\Gamma(3-\beta) \Gamma\left(\alpha+\sigma_{1}\right)}+\frac{2\left|v_{5}\right|+(2-\beta)\left|v_{2}\right|}{\Gamma(3-\beta) \Gamma\left(\alpha+\sigma_{2}\right)} \\
& +\frac{2\left|v_{8}\right|+(2-\beta)\left|v_{4}\right|}{\Gamma(3-\beta) \Gamma\left(\alpha-\beta_{1}\right)}, \\
N_{1}= & \left(1+\left|v_{3}\right|+\left|v_{7}\right|\right), \quad N_{2}=\frac{2\left|v_{7}\right|+(2-\beta)\left|v_{3}\right|}{\Gamma(3-\beta)} .
\end{aligned}
$$

In the sequel, we need the following assumptions.

(H1) The function $f: J \times \mathbb{R} \times \mathbb{R} \longrightarrow \mathbb{R}$ is continuous.

(H2)

$$
\left|f\left(t, u_{1}, v_{1}\right)-f\left(t, u_{2}, v_{2}\right)\right| \leq g(t)\left(\left|u_{1}-u_{2}\right|+\left|v_{1}-v_{2}\right|\right) .
$$

for all $u_{1}, u_{2}, v_{1}, v_{2} \in \mathbb{R} ; g \in L^{1}\left(J, \mathbb{R}_{+}\right)$and $t \in J$.

(H3) There exist a positive constant $\omega<1$ and a continuous function $\phi:[0, \infty) \longrightarrow[0, \infty)$, such that $\phi(v) \leq \omega v, \omega<\frac{1}{N_{1}+N_{2}}$ and $|g(u)-g(v)| \leq \phi(\|u-v\|)$, for all $u, v \in C(J, \mathbb{R})$.

(H4) $g(0)=0$.

(H5) There exist continuous nondecreasing function $\psi:[0, \infty) \longrightarrow(0, \infty)$ and function $p \in L^{1}\left([0,1], \mathbb{R}^{+}\right)$, such that:

$$
|f(t, u, v)| \leq p(t) \psi(|u|+|v|),
$$

for each $(t, u, v) \in[0,1] \times \mathbb{R} \times \mathbb{R}$.

(H16

$$
\sup _{r \in(0, \infty)} \frac{r}{\left(M_{1}+M_{2}\right)\|p\|_{L^{1}} \psi(r)}>\frac{1}{1-\left(N_{1}+N_{2}\right) \omega} .
$$

(H7) There exists non-negative function $p \in L^{1}\left(J, \mathbb{R}_{+}\right)$, such that:

$$
|f(t, u, v)| \leq p(t)(1+|u|+|v|),
$$

for all $u, v \in \mathbb{R}$ and $t \in J$.

Our first results is based on the Banach contraction principle.

Theorem 3.4 Assume $\left(H_{1}\right),\left(H_{2}\right)$, and $\left(H_{3}\right)$ hold. If

$$
\left.\gamma_{1}=\left(M_{1}+M_{2}\right)\|g\|_{L^{1}}+\left(N_{1}+N_{2}\right) \omega\right)<1 .
$$

holds, then the problem (1) has a unique solution on J. 
Proof Transform the problem (1) into a fixed point problem. Clearly, the fixed points of the operator $T$ defined by (10) are solutions of the problem (1).

Let $u, v \in E$ and $t \in J$, and from the definition of $T$, we can write:

$$
\begin{aligned}
& |(T u)(t)-(T v)(t)|=I_{0^{+}}^{\alpha}\left|f\left(s, u(s),{ }^{c} D_{0^{+}}^{\beta} u(s)\right)-f\left(s, v(s),{ }^{c} D_{0^{+}}^{\beta} v(s)\right)\right|(t) \\
& \quad+\left|v_{6} t^{2}-v_{1} t\right| I_{0^{+}}^{\alpha+\sigma_{1}}\left|f\left(s, u(s),{ }^{c} D_{0^{+}}^{\beta} u(s)\right)-f\left(s, v(s),{ }^{c} D_{0^{+}}^{\beta} v(s)\right)\right|\left(\eta_{1}\right) \\
& \quad+\left|v_{2} t-v_{5} t^{2}\right| I_{0^{+}}^{\alpha+\sigma_{2}}\left|f\left(s, u(s),{ }^{c} D_{0^{+}}^{\beta} u(s)\right)-f\left(s, v(s),{ }^{c} D_{0^{+}}^{\beta} v(s)\right)\right|\left(\eta_{2}\right) \\
& \quad+\left|v_{8} t^{2}-v_{4} t\right| I_{0^{+}}^{\alpha-\beta_{1}}\left|f\left(s, u(s),{ }^{c} D_{0^{+}}^{\beta} u(s)\right)-f\left(s, v(s),{ }^{c} D_{0^{+}}^{\beta} v(s)\right)\right|(1) \\
& \quad+\left|1-v_{3} t-v_{7} t^{2}\right||g(u)-g(v)| .
\end{aligned}
$$

By (H2), we can find that:

$$
\begin{aligned}
& |(T u)(t)-(T v)(t)| \leq\left(\|u-v\|_{\infty}+\left\|{ }^{c} D_{0^{+}}^{\beta} u-{ }^{c} D_{0^{+}}^{\beta} v\right\|_{\infty}\right)\left[I_{0^{+}}^{\alpha} g(s)(t)\right. \\
& \quad+\left(\left|v_{6}\right|+\left|v_{1}\right|\right) I_{0^{+}}^{\alpha+\sigma_{1}} g(s)\left(\eta_{1}\right)+\left(\left|v_{2}\right|+\left|v_{5}\right|\right) I_{0^{+}}^{\alpha+\sigma_{2}} g(s)\left(\eta_{2}\right) \\
& \left.\quad+\left(\left|v_{8}\right|+\left|v_{4}\right|\right) I_{0^{+}}^{\alpha-\beta_{1}} g(s)(1)\right] \\
& \quad+\left(1+\left|v_{3}\right|+\left|v_{7}\right|\right)|g(u)-g(v)| .
\end{aligned}
$$

According to the Lemmas 2.7, 2.8, and (H3), we have:

$$
\begin{aligned}
\|T u-T v\|_{\infty} \leq & \|u-v\|_{E}\left(\frac{1}{\Gamma(\alpha)}+\frac{\left|v_{6}\right|+\left|v_{1}\right|}{\Gamma\left(\alpha+\sigma_{1}\right)}+\frac{\left|v_{2}\right|+\left|v_{5}\right|}{\Gamma\left(\alpha+\sigma_{2}\right)}+\frac{\left|v_{8}\right|+\left|v_{4}\right|}{\Gamma\left(\alpha-\beta_{1}\right)}\right)\|g\|_{L^{1}} \\
& +\left(1+\left|v_{3}\right|+\left|v_{7}\right|\right) \omega\|u-v\|_{\infty} .
\end{aligned}
$$

Thus:

$$
\|T u-T v\|_{\infty} \leq\left(M_{1}\|g\|_{L^{1}}+N_{1} \omega\right)\|u-v\|_{E} .
$$

Observe that:

$$
\begin{aligned}
{ }^{c} D_{0^{+}}^{\beta} T u(t)= & I_{0^{+}}^{\alpha-\beta} f\left(s, u(s),{ }^{c} D_{0^{+}}^{\beta} u(s)\right)(t) \\
& +\frac{2 v_{6} t^{2-\beta}+(2-\beta) v_{1} t^{1-\beta}}{\Gamma(3-\beta)} I_{0^{+}}^{\alpha+\sigma_{1}} f\left(s, u(s),{ }^{c} D_{0^{+}}^{\beta} u(s)\right)\left(\eta_{1}\right) \\
& +\frac{2 v_{5} t^{2-\beta}+(2-\beta) v_{2} t^{1-\beta}}{\Gamma(3-\beta)} I_{0^{+}}^{\alpha+\sigma_{2}} f\left(s, u(s),{ }^{c} D_{0^{+}}^{\beta} u(s)\right)\left(\eta_{2}\right) \\
& +\frac{2 v_{8} t^{2-\beta}+(2-\beta) v_{4} t^{1-\beta}}{\Gamma(3-\beta)} I_{0^{+}}^{\alpha-\beta_{1}} f\left(s, u(s),{ }^{c} D_{0^{+}}^{\beta} u(s)\right)(1) \\
& +\frac{2 v_{7} t^{2-\beta}+(2-\beta) v_{3} t^{1-\beta}}{\Gamma(3-\beta)} g(u),
\end{aligned}
$$

using similar techniques as to get (19), it yields:

$$
\left\|{ }^{c} D_{0^{+}}^{\beta} T u-{ }^{c} D_{0^{+}}^{\beta} T v\right\|_{\infty} \leq\left(M_{2}\|g\|_{L^{1}}+N_{2} \omega\right)\|u-v\|_{E} .
$$

Combining (19) and (20), we get:

$$
\|T u-T v\|_{E} \leq\left(\left(M_{1}+M_{2}\right)\|g\|_{L^{1}}+\left(N_{1}+N_{2}\right) \omega\right)\|u-v\|_{E} .
$$

Therefore, we have:

$$
\|T u-T v\|_{E} \leq \gamma_{1}\|u-v\|_{E} .
$$

In view of the given condition $\left.\gamma_{1}=\left(M_{1}+M_{2}\right)\|g\|_{L^{1}}+\left(N_{1}+N_{2}\right) \omega\right)<1$, it follows that the mapping $T$ is a contraction. Hence, by the Banach fixed point theorem, $T$ has a unique fixed point which is a unique solution of Problem (1). This completes the proof. 
Our next result relies on a fixed point theorem of O'Regan [22]

Lemma 3.5 Denote by $U$ an open set in a closed, convex set $C$ of a Banach space E. Assume $0 \in U$. Also assume that $T(\bar{U})$ is bounded and that $F: \bar{U} \longrightarrow C$ is given by $T=T_{1}+T_{2}$, in which $T_{1}: \bar{U} \longrightarrow E$ is continuous and completely continuous and $T_{2}: \bar{U} \longrightarrow E$ is a nonlinear contraction (i.e., there exists a non-negative nondecreasing function $\phi:[0, \infty) \longrightarrow[0, \infty)$ satisfying $\phi(z)<z$ for $z>0$, such that $\left\|T_{2}(u)-T_{2}(v)\right\| \leq \phi(\|u-v\|)$ for all $\left.u, v \in \bar{U}\right)$. Then, either

(c1) $T$ has a fixed point in $\bar{U}$, or

(c2) there exists $u \in \partial U$ and $\lambda \in(0,1)$, such that $u=\lambda T u$, where $\bar{U}$ and $\partial U$, respectively, represent the closure and boundary of $U$.

Let

$$
B_{r}=\left\{u \in E:\|u\|_{E}<r\right\}
$$

and denote the maximum number by:

$$
M_{r}=\max \{|f(t, u, v)|:(t, u, v) \in J \times[-r, r] \times[-r, r]\} .
$$

Theorem 3.6 Suppose that (H1),(H3)-(H6) are satisfied. Then, the problem (1) has at least one solution on $J$.

Proof From (H6), there exists a number $r_{0}>0$, such that:

$$
\frac{r_{0}}{\left(M_{1}+M_{2}\right)\|p\|_{L^{1}} \psi\left(r_{0}\right)}>\frac{1}{1-\left(N_{1}+N_{2}\right) \omega} .
$$

We shall prove that $T_{1}$ and $T_{2}$ defined by (11) and (12), respectively, satisfy the conditions of Lemma 3.5. We split the proof into several steps.

Step 1 : The operator $T_{1}$ is completely continuous. It is clear that $T_{1}$ is continuous, since $f$ is continuous.

Next, we shall prove that the operator $T_{1}$ maps bounded sets into bounded sets in $E$. For $u \in \bar{B}_{r_{0}}$ and for each $t \in J$, we have:

$$
\begin{aligned}
\left|\left(T_{1} u\right)(t)\right| \leq & I_{0^{+}}^{\alpha}\left|f\left(s, u(s),{ }^{c} D_{0^{+}}^{\beta} u(s)\right)\right|(t) \\
& +\left(\left|v_{6}\right|+\left|v_{1}\right|\right) I_{0^{+}}^{\alpha+\sigma_{1}}\left|f\left(s, u(s),{ }^{c} D_{0^{+}}^{\beta} u(s)\right)\right|\left(\eta_{1}\right) \\
& +\left(\left|v_{2}\right|+\left|v_{5}\right|\right) I_{0^{+}}^{\alpha+\sigma_{2}}\left|f\left(s, u(s),{ }^{c} D_{0^{+}}^{\beta} u(s)\right)\right|\left(\eta_{2}\right) \\
& +\left(\left|v_{8}\right|+\left|v_{4}\right|\right) I_{0^{+}}^{\alpha-\beta_{1}}\left|f\left(s, u(s),{ }^{c} D_{0^{+}}^{\beta} u(s)\right)\right|(1) .
\end{aligned}
$$

In view of (15), we obtain:

$$
\begin{aligned}
\left|\left(T_{1} u\right)(t)\right| \leq & \psi(\|u\|)\left\{I_{0^{+}}^{\alpha} p(s)(t)+\left(\left|v_{6}\right|+\left|v_{1}\right|\right) I_{0^{+}}^{\alpha+\sigma_{1}} p(s)\left(\eta_{1}\right)\right. \\
& \left.+\left(\left|v_{2}\right|+\left|v_{5}\right|\right) I_{0^{+}}^{\alpha+\sigma_{2}} p(s)\left(\eta_{2}\right)+\left(\left|v_{8}\right|+\left|v_{4}\right|\right) I_{0^{+}}^{\alpha-\beta_{1}} p(s)(1)\right\} .
\end{aligned}
$$

By Lemmas (2.7), ( 2.8), we obtain:

$$
\left|\left(T_{1} u\right)(t)\right| \leq M_{1}\|p\|_{L^{1}} \psi_{1}\left(r_{0}\right) .
$$

Thus:

$$
\left\|T_{1} u\right\|_{\infty} \leq M_{1}\|p\|_{L^{1}} \psi_{1}\left(r_{0}\right) .
$$

With the same arguments as in (22), we get:

$$
\left\|{ }^{c} D_{0^{+}}^{\beta} T_{1} u\right\|_{\infty} \leq M_{2}\|p\|_{L^{1}} \psi_{1}\left(r_{0}\right) .
$$


Combining (22) with (23), we get:

$$
\left\|T_{1} u\right\|_{E} \leq\left(M_{1}+M_{2}\right)\|p\|_{L^{1}} \psi_{1}\left(r_{0}\right),
$$

which shows that $T_{1}$ is uniform bounded.

Now, we will prove that $T_{1}\left(\bar{B}_{r_{0}}\right)$ is equi-continuous. Let $t_{1}, t_{2} \in[0,1], t_{1}<t_{2}, u \in \bar{B}_{r_{0}}$ :

$$
\begin{aligned}
& \left|\left(T_{1} u\right)\left(t_{2}\right)-\left(T_{1} u\right)\left(t_{1}\right)\right| \\
& \leq \frac{1}{\Gamma(\alpha)} \int_{0}^{t_{1}}\left[\left(t_{2}-s\right)^{\alpha-1}-\left(t_{1}-s\right)^{\alpha-1}\right]\left|f\left(s, u(s),{ }^{c} D_{0^{+}}^{\beta} u(s)\right)\right| \mathrm{d} s \\
& \quad+\frac{1}{\Gamma(\alpha)} \int_{t_{1}}^{t_{2}}\left(t_{2}-s\right)^{\alpha-1}\left|f\left(s, u(s),{ }^{c} D_{0^{+}}^{\beta} u(s)\right)\right| \mathrm{d} s \\
& \quad+\left(\left|v_{6}\right|\left(t_{2}^{2}-t_{1}^{2}\right)+\left|v_{1}\right|\left(t_{2}-t_{1}\right)\right) I_{0^{+}}^{\alpha+\sigma_{1}}\left|f\left(s, u(s),{ }^{c} D_{0^{+}}^{\beta} u(s)\right)\right|\left(\eta_{1}\right) \\
& \quad+\left(\left|v_{2}\right|\left(t_{2}-t_{1}\right)+\left|v_{5}\right|\left(t_{2}^{2}-t_{1}^{2}\right)\right) I_{0^{+}}^{\alpha+\sigma_{2}}\left|f\left(s, u(s),{ }^{c} D_{0^{+}}^{\beta} u(s)\right)\right|\left(\eta_{2}\right) \\
& \quad+\left(\left|v_{8}\right|\left(t_{2}^{2}-t_{1}^{2}\right)+\left|v_{4}\right|\left(t_{2}-t_{1}\right)\right) I_{0^{+}}^{\alpha-\beta_{1}}\left|f\left(s, u(s),{ }^{c} D_{0^{+}}^{\beta} u(s)\right)\right|(1) .
\end{aligned}
$$

Some computations give:

$$
\begin{aligned}
\left|\left(T_{1} u\right)\left(t_{2}\right)-\left(T_{1} u\right)\left(t_{1}\right)\right| \leq & M_{r}\left[\frac{1}{\Gamma(\alpha)}+\frac{2\left|v_{6}\right|+\left|v_{1}\right|}{\Gamma\left(\alpha+\sigma_{1}+1\right)} \eta_{1}^{\alpha+\sigma_{1}} \frac{2\left|v_{2}\right|+\left|v_{5}\right|}{\Gamma\left(\alpha+\sigma_{2}+1\right)} \eta_{2}^{\alpha+\sigma_{2}}\right. \\
& \left.+\frac{2\left|v_{8}\right|+\left|v_{4}\right|}{\Gamma\left(\alpha-\beta_{1}+1\right)}\right]\left(t_{2}-t_{1}\right) .
\end{aligned}
$$

In a like manner:

$$
\begin{gathered}
\left|{ }^{c} D_{0^{+}}^{\beta}\left(T_{1} u\right)\left(t_{2}\right)-{ }^{c} D_{0^{+}}^{\beta}\left(T_{1} u\right)\left(t_{1}\right)\right| \leq M_{r}\left\{\frac{t_{2}^{\alpha-\beta}-t_{1}^{\alpha-\beta}}{\Gamma(\alpha-\beta+1)}\right. \\
+\frac{2\left|v_{6}\right|\left|t_{2}^{2-\beta}-t_{1}^{2-\beta}\right|+(2-\beta)\left|v_{1}\right|\left|t_{2}^{1-\beta}-t_{1}^{1-\beta}\right|}{\Gamma(3-\beta) \Gamma\left(\alpha+\sigma_{1}+1\right)} \eta_{1}^{\alpha+\sigma_{1}} \\
+\frac{2\left|v_{5}\right|\left|t_{2}^{2-\beta}-t_{1}^{2-\beta}\right|+(2-\beta)\left|v_{2}\right|\left|t_{2}^{1-\beta}-t_{1}^{1-\beta}\right|}{\Gamma(3-\beta) \Gamma\left(\alpha+\sigma_{2}+1\right)} \eta_{2}^{\alpha+\sigma_{2}} \\
\left.+\frac{2\left|v_{8}\right|\left|t_{2}^{2-\beta}-t_{1}^{2-\beta}\right|+(2-\beta)\left|v_{4}\right|\left|t_{2}^{1-\beta}-t_{1}^{1-\beta}\right|}{\Gamma(3-\beta) \Gamma\left(\alpha-\beta_{1}+1\right)}\right\} .
\end{gathered}
$$

In consequence, we obtain:

$$
\sup _{u \in \bar{B}_{r_{0}}}\left|\left(T_{1} u\right)\left(t_{2}\right)-\left(T_{1} u\right)\left(t_{1}\right)\right|+\sup _{u \in \bar{B}_{r_{0}}}\left|{ }^{c} D_{0^{+}}^{\beta}\left(T_{1} u\right)\left(t_{2}\right)-{ }^{c} D_{0^{+}}^{\beta}\left(T_{1} u\right)\left(t_{1}\right)\right| \rightarrow 0,
$$

as $t_{1} \rightarrow t_{2}$ independent of $u \in \bar{B}_{r_{0}}$. Therefore, the operator $T_{1}$ is equi-continuous and uniformly bounded. Hence, by the Arzelá-Ascoli theorem, it follows that $T_{1}\left(\bar{B}_{r_{0}}\right)$ is relatively compact in $E$.

Step 2 : The operator $T_{2}: \bar{B}_{r_{0}} \longrightarrow E$ is contractive. Let $u, v \in$ and $t \in J$ by (H3), we can show that:

$$
\left\|T_{2}(u)-T_{2}(v)\right\|_{E} \leq\left(N_{1}+N_{2}\right) \omega\|u-v\|_{E},
$$

which, in view of (H3), implies that $T_{2}$ is a contraction.

Step 3: The set $T_{2}\left(\bar{B}_{r_{0}}\right)$ is bounded. For $u \in \bar{B}_{r_{0}}$ and for each $t \in J$, and by (H3) and (H4), we obtain:

$$
\left\|T_{2} u\right\|_{E} \leq\left(N_{1}+N_{2}\right) \omega r_{0} .
$$


Hence, $T_{2}\left(\bar{B}_{r_{0}}\right)$ is bounded.

Step 4 : Finally, we show that the case (c2) in Lemma 3.5 does not occur. To this end, we suppose that (c2) holds. Let $u \in \partial B_{r_{0}}$, and then, $u=\lambda T u$, for some $0<\lambda<1$. Therefore, we have $\|u\|_{E}=r_{0}$, and:

$$
\begin{aligned}
u(t)= & \lambda\left\{I_{0^{+}}^{\alpha} f\left(s, u(s),{ }^{c} D_{0^{+}}^{\beta} u(s)\right)(t)\right. \\
& +\left(v_{6} t^{2}-v_{1} t\right) I_{0^{+}}^{\alpha+\sigma_{1}} f\left(s, u(s),{ }^{c} D_{0^{+}}^{\beta} u(s)\right)\left(\eta_{1}\right) \\
& +\left(v_{2} t-v_{5} t^{2}\right) I_{0^{+}}^{\alpha+\sigma_{2}} f\left(s, u(s),{ }^{c} D_{0^{+}}^{\beta} u(s)\right)\left(\eta_{2}\right) \\
& +\left(v_{8} t^{2}-v_{4} t\right) I_{0^{+}}^{\alpha-\beta_{1}} f\left(s, u(s),{ }^{c} D_{0^{+}}^{\beta} u(s)\right)(1) \\
& \left.+\left(1-v_{3} t-v_{7} t^{2}\right) g(u)\right\} .
\end{aligned}
$$

With hypotheses (H3)-(H6), we can write:

$$
r_{0} \leq\left(M_{1}+M_{2}\right)\|p\|_{L^{1}} \psi\left(r_{0}\right)+\left(N_{1}+N_{2}\right) \omega r_{0},
$$

which implies that:

$$
\frac{r_{0}}{\left(M_{1}+M_{2}\right)\|p\|_{L^{1}} \psi\left(r_{0}\right)} \leq \frac{1}{1-\left(N_{1}+N_{2}\right) \omega},
$$

which contradicts (16). Consequently, we have proved that the operators $T_{1}$ and $T_{2}$ satisfy all the conditions in Lemma 3.5. Hence, the operator $T$ has at least one fixed point $u \in \bar{B}_{r_{0}}$, which is the solution of the boundary value problem (1).

Our next result relies on the following fixed point theorem due to Burton and Kirk [8]

Theorem 3.7 Let $E$ be a Banach space, and $A, B: E \longrightarrow E$ be two operators, such that $A$ is a contraction and $B$ is completely continuous. Then, either

- the operator equation $u=A(u)+B(u)$ has a solution, or

- the set $\Omega=\left\{u \in E: \lambda A\left(\frac{u}{\lambda}\right)+\lambda B(u)=u\right\}$ is unbounded for $\lambda \in(0,1)$.

Then, there exists $z \in U$, such that $z=A z+B z$.

Theorem 3.8 Assume that (H1), (H3), (H4), and (H7) hold. In addition, we suppose that:

$$
\left(M_{1}+M_{2}\right)\|p\|_{L^{1}}+\left(N_{1}+N_{2}\right) \omega<1 .
$$

Then, the fractional boundary value problem (1) has at least one solution on J.

Proof We define the map $T: E \longrightarrow E$ by:

$$
T u(t)=T_{1} u(t)+T_{2} u(t), t \in J,
$$

where $T_{1}$ and $T_{2}$ are defined by (11) and (12), respectively. Notice that Problem (1) is equivalent to the fixed point problem $T(u)=u$. We shall prove that $T_{1}$ and $T_{2}$ satisfy the conditions of Theorem 3.7. For clarity, we will divide the remain of the proof into several steps.

Step 1: $T_{1}$ is continuous. The continuity of $f$ implies that the operator $T_{1}$ is continuous.

Step 2: The operator $T_{1}$ maps bounded sets into bounded sets in $E$. In a similar manner as previous proof of Theorem 3.6, we can find that:

$$
\left\|T_{1} u\right\|_{E} \leq\left(M_{1}+M_{2}\right)\|p\|_{L^{1}}\left(1+\|u\|_{E}\right) .
$$

Step 3: The operator $T_{1}$ maps bounded sets into equi-continuous sets. By a discussion similar to that of Theorem 3.6, we can get:

$$
\left|\left(T_{1} u\right)\left(t_{2}\right)-\left(T_{1} u\right)\left(t_{1}\right)\right| \rightarrow 0 .
$$

And

$$
\left|{ }^{c} D_{0^{+}}^{\beta} T_{1} y\left(t_{2}\right)-{ }^{c} D_{0^{+}}^{\beta} T_{1} y\left(t_{1}\right)\right| \rightarrow 0
$$


as $t_{2} \rightarrow t_{1}$. This implies that:

$$
\left\|(T u)\left(t_{2}\right)-(T u)\left(t_{1}\right)\right\|_{E} \rightarrow 0, \text { as } t_{2} \rightarrow t_{1} .
$$

Step 4: The operator $T_{2}$ is a contraction. This was established in Step 2 of Theorem 3.6.

Step 5: A priori bounds on solutions. Now, it remains to show that the set $\Omega=\left\{u \in E: \lambda T_{2}\left(\frac{u}{\lambda}\right)+\lambda T_{1}(u)=u\right\}$ is unbounded for $\lambda \in(0,1)$. Let $\lambda \in(0,1)$, and $u \in E$ be a solution of the integral equation:

$$
\begin{aligned}
u(t)= & \lambda\left\{I_{0^{+}}^{\alpha} f\left(s, u(s),{ }^{c} D_{0^{+}}^{\beta} u(s)\right)(t)\right. \\
& +\left(v_{6} t^{2}-v_{1} t\right) I_{0^{+}}^{\alpha+\sigma_{1}} f\left(s, u(s),{ }^{c} D_{0^{+}}^{\beta} u(s)\right)\left(\eta_{1}\right) \\
& +\left(v_{2} t-v_{5} t^{2}\right) I_{0^{+}}^{\alpha+\sigma_{2}} f\left(s, u(s),{ }^{c} D_{0^{+}}^{\beta} u(s)\right)\left(\eta_{2}\right) \\
& +\left(v_{8} t^{2}-v_{4} t\right) I_{0^{+}}^{\alpha-\beta_{1}} f\left(s, u(s),{ }^{c} D_{0^{+}}^{\beta} u(s)\right)(1) \\
& \left.+\left(1-v_{3} t-v_{7} t^{2}\right) g\left(\frac{y}{\lambda}\right)\right\} .
\end{aligned}
$$

This implies by (H3), (H4), and (H7) that, for each $t \in J$, we have:

$$
\begin{aligned}
|u(t)| \leq & I_{0^{+}}^{\alpha}\left|f\left(s, u(s),{ }^{c} D_{0^{+}}^{\beta} u(s)\right)\right|(t) \\
& +\left(\left|v_{6}\right|+\left|v_{1}\right|\right) I_{0^{+}}^{\alpha+\sigma_{1}}\left|f\left(s, u(s),{ }^{c} D_{0^{+}}^{\beta} u(s)\right)\right|\left(\eta_{1}\right) \\
& +\left(\left|v_{2}\right|+\left|v_{5}\right|\right) I_{0^{+}}^{\alpha+\sigma_{2}}\left|f\left(s, u(s),{ }^{c} D_{0^{+}}^{\beta} u(s)\right)\right|\left(\eta_{2}\right) \\
& +\left(\left|v_{8}\right|+\left|v_{4}\right|\right) I_{0^{+}}^{\alpha-\beta_{1}}\left|f\left(s, u(s),{ }^{c} D_{0^{+}}^{\beta} u(s)\right)\right|(1) \\
& +\left(1+\left|v_{3}\right|+\left|v_{7}\right|\right) \mid g\left(\frac{u(t)}{\lambda}\right) \\
\leq & \left(1+\|u\|_{E}\right)\left(I_{0^{+}}^{\alpha} p(s)(t)+\left(\left|v_{6}\right|+\left|v_{1}\right|\right) I_{0^{+}}^{\alpha+\sigma_{1}} p(s)\left(\eta_{1}\right)\right. \\
& \left.+\left(\left|v_{2}\right|+\left|v_{5}\right|\right) I_{0^{+}}^{\alpha+\sigma_{2}} p(s)\left(\eta_{2}\right)+\left(\left|v_{8}\right|+\left|v_{4}\right|\right) I_{0^{+}}^{\alpha-\beta_{1}} p(s)(1)\right) \\
& +\left(1+\left|v_{3}\right|+\left|v_{7}\right|\right) \omega\|u\| .
\end{aligned}
$$

According to the Lemmas 2.7 and 2.8, we have:

$$
\|u\|_{\infty} \leq M_{1}\|p\|_{L^{1}}\left(1+\|u\|_{E}\right)+N_{1} \omega\|u\|,
$$

for all $t \in J$. In a similar manner, we obtain:

$$
\left\|{ }^{c} D_{0^{+}}^{\beta} u\right\|_{\infty} \leq M_{2}\|p\|_{L^{1}}\left(1+\|u\|_{E}\right)+N_{2} \omega\|u\| .
$$

Combining (27) with (28), we get:

$$
\|u\|_{E} \leq \frac{\left(M_{1}+M_{2}\right)\|p\|_{L^{1}}}{1-\left(\left(M_{1}+M_{2}\right)\|p\|_{L^{1}}+\left(N_{1}+N_{2}\right) \omega\right)} .
$$

This shows that the set $\Omega$ is bounded, since $\left(M_{1}+M_{2}\right)\|p\|_{L^{1}}+\left(N_{1}+N_{2}\right) \omega<1$. Hence, $T$ has a fixed point by Theorem 3.7, and consequently, the problem (1) has a solution. This completes the proof. 


\section{An example}

Example 4.1 Let us consider Problem (1) with specific data:

$$
\begin{gathered}
\alpha=\frac{5}{2}, \beta=\frac{1}{2}, \beta_{1}=\frac{1}{4}, \\
a=1, b=2, \sigma_{1}=\frac{5}{2}, \\
\sigma_{2}=\frac{7}{2}, \eta_{1}=\frac{1}{4}, \eta_{2}=\frac{1}{2} .
\end{gathered}
$$

Using the given values of the parameters in (6) and (14), by the Matlab program, we find that:

$$
\begin{aligned}
v_{1} & =-1.0062, v_{2}=0.0027, v_{3}=-0.0535, \\
v_{4} & =0.0014, v_{5}=-1.6060, v_{6}=-0.8803, \\
v_{7} & =0.0457, v_{8}=-0.8030, M_{1}=1.5542, \\
M_{2} & =2.1903, M_{1}+M_{2}=3.7445, N_{1}=1.0993, \\
N_{2} & =0.1292, N_{1}+N_{2}=1.2285, \Delta=0.6179, .
\end{aligned}
$$

To illustrate Theorem 3.4, we take:

$$
\begin{aligned}
f\left(t, u(t),{ }^{c} D_{0^{+}}^{\frac{1}{4}} u(t)\right) & =\frac{(1-t)^{2}}{10}\left(\frac{1}{2}\left(x(t)+\sqrt{x^{2}(t)+1}\right)\right. \\
& \left.+\frac{\left|{ }^{c} D_{0^{+}}^{\frac{1}{4}} u(t)\right|}{1+\left|{ }^{c} D_{0^{+}}^{\frac{1}{4}} u(t)\right|}\right)+e^{-t}, \\
g(u) & =\frac{1}{10}(1-\cos u),,
\end{aligned}
$$

in (1) and note that:

$$
\begin{aligned}
\left|f(t, u, v)-f\left(t, u_{1}, v_{1}\right)\right| \leq & \frac{(1-t)^{2}}{10}\left(\left|\frac{1}{2}\left(u-u_{1}+\sqrt{1+u^{2}}-\sqrt{1+u_{1}^{2}}\right)\right|\right. \\
& \left.+\left|\frac{|v|}{1+|v|}-\frac{\left|v_{1}\right|}{1+\left|v_{1}\right|}\right|\right) \\
\leq & \frac{(1-t)^{2}}{10}\left(\left|\frac{1}{2}\left(u-u_{1}\right)\left(1+\frac{u+u_{1}}{\sqrt{1+u^{2}}+\sqrt{1+u_{1}^{2}}}\right)\right|\right. \\
& \left.+\frac{\left|v-v_{1}\right|}{(1+|v|)\left(1+\left|v_{1}\right|\right)}\right) \\
\leq & \frac{(1-t)^{2}}{10}\left(\left|u-u_{1}\right|+\left|v-v_{1}\right|\right)
\end{aligned}
$$

for each $(t, u, v) \in[0,1] \times \mathbb{R} \times \mathbb{R}$ and

$$
|g(u)-g(v)| \leq \frac{1}{10}|u-v|,
$$

for each $(u, v) \in \mathbb{R} \times \mathbb{R}$.

Here, $\omega=\frac{1}{10}, g(t)=\frac{(1-t)^{2}}{10}$ with $\|g\|_{L^{1}}=\frac{1}{30}$. Using the values of $M_{1}+M_{2}$ and $N_{1}+N_{2}$ given by (30), we find that $\gamma_{1}=\left(M_{1}+M_{2}\right)\|g\|_{L^{1}}+\left(N_{1}+N_{2}\right) \omega=0.2477<1$. As all the conditions of Theorem 3.4 are satisfied, therefore, there exists a unique solution of Problem (1) with the data (29) and (31) on $J$. 


\section{Conclusion}

We have established the existence and uniqueness of solutions for fractional boundary value problem under more general assumptions. Using the techniques of fixed point theorems such as Banach contraction principle, O'Regan fixed point theorem, and Burton and Kirk fixed point theorem, we establish the main results of the paper. Finally, we conclude the paper with an example to make our results clear.

Acknowledgements We are thankful to the referees for their carefully reading and constructive comments to improve the overall quality of this work.

Open Access This article is licensed under a Creative Commons Attribution 4.0 International License, which permits use, sharing, adaptation, distribution and reproduction in any medium or format, as long as you give appropriate credit to the original author(s) and the source, provide a link to the Creative Commons licence, and indicate if changes were made. The images or other third party material in this article are included in the article's Creative Commons licence, unless indicated otherwise in a credit line to the material. If material is not included in the article's Creative Commons licence and your intended use is not permitted by statutory regulation or exceeds the permitted use, you will need to obtain permission directly from the copyright holder. To view a copy of this licence, visit http://creativecommons.org/licenses/by/4.0/.

\section{References}

1. Abbas, S.; Albarakati, W.; Benchohra, M.; Trujillo, J.J.: Ulam stabilities for partial Hadamard fractional integral equations. Arab. J. Math. 5, 1-7 (2016)

2. Ahmad, B.; Ntouyas, S.K.; Assolami, A.: Caputo type fractional differential equations with nonlocal Riemann-Liouville integral boundary conditions. J. Appl. Math. Comput. 41, 339-350 (2013)

3. Ahmadkhanlu, A.: Existence and uniquensess for a class of fractional differential Equations with an integral fractional boundary condition. Filomat 31(5), 1241-1246 (2017)

4. Benchohra, M.; Hamani, S.; Ntouyas, S.K.: Boundary value problems for differential equations with fractional order and nonlocal conditions. Nonlinear Anal. 71, 2391-2396 (2009)

5. Benchohra, M.; Souid, M.: Integrable solutions for implicit fractional order differential equations. TJMM 6(2), 101-107 (2014)

6. Benchohra, M.; Lazreg, J.E.: Existence results for nonlinear implicit fractional differential equations. Surv. Math. Appl. 9, 79-92 (2014)

7. Benchohra, M.; Berhoun, F.; N'Guèrèkata, G.M.: Bounded solutions for fractional order differential equations on the half-line. Bull. Math. Anal. Appl 146(4), 62-71 (2012)

8. Burton, T.A.; Kirk, C.: A fixed point theorem of Krasnoselskii-Schaefer type. Math. Nachr. 189, 23-31 (1998)

9. Cabada, A.; Wang, G.: Positive solutions of nonlinear fractional differential equations with integral boundary value conditions. J. Math. Anal. 389, 403-411 (2012)

10. El-Sayed, A.M.A.; Bin-Taher, E.O.: Positive solutions for a nonlocal multipoint bounday-value problem of fractional and second order. Electron. J. Differ. Equ. 2013(64), 1-8 (2014)

11. El-Sayed, A.M.A.; Bin-Taher, E.O.: Positive solutions for a nonlocal boundaryvalue problem of a class of arbitrary (fractional) orders differential equations. Int. J. Nonlinear Sci. 14(4), 398-404 (2012)

12. Guezane-Lakoud, A.; Khaldi, R.: Solvability of a fractional boundary value problem with integral condition. Nonlinear Anal. 75, 2692-2700 (2012)

13. Guezane-Lakoud, A.; Bensebaa, S.: Solvability of a fractional boundary value problem with fractional derivative condition. Arab. J. Math. 3, 39-48 (2014)

14. Houas, M.; Benbachir, M.: Existence solutions for three point boundary value problem for differential equations. JFCA 6(1), 160-174 (2015)

15. Houas, M.; Dahmani, Z.: On existence of solutions for fractional differential equations with nonlocal multi-point boundary conditions. Lobachevskii J. Math. 37(2), 120-127 (2016)

16. Kilbas, A.A.; Srivastava, H.M.; Trujillo, J.J.: Theory and applications of fractional differential equations. In: North-Holland Mathematics Studies, vol. 204. Elsevier, Amsterdam (2006)

17. Liu, Y.: Solvability of impulsive periodic boundary value problems for higher order fractional differential equations. Arab. J. Math. 5, 195-214 (2016)

18. Matar, M.; Trujillo, J.J.: Existence of local solutions for differential equations with arbitrary fractional order. Arab. J. Math. 5, 215-224 (2016)

19. Miller, K.S.; Ross, B.: An Introduction to Fractional Calculus and Fractional Differential Equations. Wiley, New YorK (1993)

20. Nieto, J.J.; Ouahab, A.; Venktesh, V.: Implicit fractional differential equations via the Liouville-Caputo derivative. Mathematics 3, 398-411 (2015)

21. Ntouyas. S. K.: Boundary value problems for nonlinear fractional differential equations and inclusions with nonlocal and fractional integral boundary conditions. Opuscula Math. 33(1), 117-138 (2013)

22. O'Regan, D.: Fixed-point theory for the sum of two operators. Appl. Math. Lett. 9, 1-8 (1996)

23. Podlubny, I.: Fractional Differential Equations. Academic Press, San Diego (1993)

24. Samko, G.; Kilbas, A.A.; Marichev, O.I.: Fractional Integral and Derivative; Theory and Applications. Gordon and Breach, Yverdon (1993)

25. Su, C.M.; Sun, J.P.; Zhao, Y.H.: Existence and uniqueness of solutions for BVP of nonlinear fractional differential equation. Hindawi Int. J. Differ. Equ. 2017(4683581), 1-7 (2017) 
26. Su, X.: Boundary value problem for a coupled system of nonlinear fractional differential equations. Appl. Math. Lett. 22, 64-69 (2009)

27. Yan, R.; Sun, S.; Lu, H.; Zhao, Y.: Existence of solutions for fractional differential equations with integral boundary condition. Adv. Differ. Equ. 2014, 25 (2014)

Publisher's Note Springer Nature remains neutral with regard to jurisdictional claims in published maps and institutional affiliations. 